\title{
La théorie du petit monde : un test sur le graphe des administrateurs des compagnies d'assurance en France en 1881
}

Jérôme Maati et Christian Rietsch

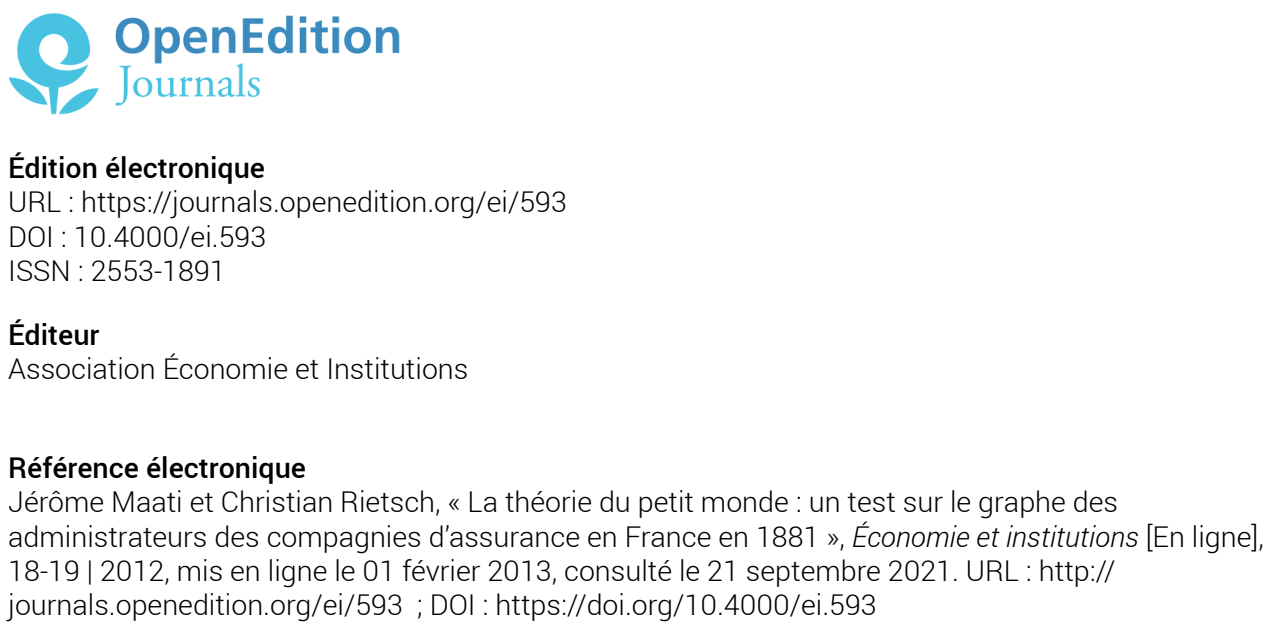

Ce document a été généré automatiquement le 21 septembre 2021.

Revue Économie et institutions 


\title{
La théorie du petit monde : un test sur le graphe des administrateurs des compagnies d'assurance en France en 1881
}

\author{
Jérôme Maati et Christian Rietsch
}

Les réseaux d'administrateurs de sociétés émanant des contacts sociaux qu'ils entretiennent au travers de l'exercice de leur mandat portent à questionnement. L'analyse la structure du graphe des administrateurs des principales compagnies d'assurance en France en 1881 vise à étudier l'existence, dans une perspective historique, d'une structure en réseau dans un secteur de création relativement récente et dont la base d'activité vient de s'élargir. Si tel est le cas, quel type d'architecture domine? Le monde de l'assurance a une structure spécifique tout au long du XIX ${ }^{\mathrm{e}}$ siècle : modalités de libération du capital, de cotation des actions, de transfert des actions, de tenue des assemblées générales et de choix des administrateurs. Sur ce dernier point, le problème se pose avec d'autant plus d'acuité qu'une décision du Conseil d'État de 1826 oblige les compagnies d'assurance à séparer les risques, ce qui conduit l'ensemble du secteur à une structure de groupe (plusieurs compagnies sœurs qui couvrent chacune un risque) à une époque qui ne connaît pas la notion de holding. D'où la question du partage des administrateurs. En outre, certaines banques ont investi dans plusieurs compagnies d'assurance rivales avec les conflits d'intérêts potentiels sous-jacents. Enfin, le problème d'une tarification des risques se pose et fait l'objet de plusieurs prises de contact entre compagnies concurrentes. Outre Atlantique à la même époque, le législateur s'inquiète déjà de la présence de liens humains permettant de connecter certains conseils d'administration. La perspective historique est-elle semblable dans les deux cas? Si le réseau réel ne peut se distinguer significativement d'un réseau issu d'un processus aléatoire d'allocation des liens, cela signifie que même si des stratégies locales peuvent être engagées, elles n'ont pas de conséquences sur la structure globale du réseau. 
2 Cette étude montre d'abord que la répartition des mandats entre les administrateurs est très inégale, près de $60 \%$ d'entre eux ne disposant que d'un mandat en dépit des parentés entre compagnies provenant de la structure en groupe du secteur. En outre, la présence d'administrateurs multi-mandats fait émerger un réseau formé de plus de $70 \%$ de la population des administrateurs. Ces deux caractéristiques structurelles conduisent à l'émergence de personnalités centrales. La structure "petit monde" du réseau au sens de Watts (1999) est vérifiée. Par ailleurs, à partir de la méthodologie proposée par Newman et al. (2001) fondée sur les fonctions génératrices des distributions de probabilités observées, des réseaux aléatoires sont construits afin de permettre une approximation de la structure réelle des réseaux. Ils montrent que la structure " petit monde » s'explique en grande partie par l'organisation du réseau en conseils d'administration. Néanmoins, les différences de valeurs entre les paramètres du graphe aléatoire et ceux du graphe réel laissent supposer l'existence de forces non aléatoires en œuvre.

3 La première partie de cet article dresse une revue de la littérature portant sur l'analyse des réseaux d'administrateurs. Après une description générale de la population étudiée, le phénomène du petit monde est testé sur les données. Enfin, la construction d'un réseau aléatoire équivalent au réseau réel permet d'expliquer la structure " petit monde ».

\section{Revue de la littérature}

4 De nombreuses législations imposent la présence d'un conseil d'administration dans les sociétés (U.S.A., France, Grande-Bretagne, Japon, etc.). Aussi, la recherche académique s'est questionnée sur son efficience en s'interrogeant notamment sur le rôle des liens humains entre les conseils d'administration. Un tel lien est établi entre deux conseils lorsqu'un individu y siège simultanément. En effet, l'appartenance d'un administrateur à un conseil n'est pas exclusive. Un administrateur peut siéger dans plusieurs conseils même si les législations prévoient souvent un nombre maximum de mandats pouvant être détenu simultanément par une même personne physique.

5 La création d'un tel lien pourrait être qualifiée d'événement marginal. Toutefois, les liens humains entre conseils d'administration ont été analysés dès la fin du xix ${ }^{e}$ siècle par le congrès américain et ont ensuite donné naissance à une abondante littérature dont l'essor est favorisé par le caractère public de l'information relative à l'appartenance des individus à un conseil d'administration.

6 Plusieurs explications non exclusives, adoptant une perspective inter-organisationnelle, sociale ou individuelle, ont été avancées et peuvent être regroupées, à l'instar de Mizruchi (1996), en quatre thèmes : la collusion, le contrôle des ressources, le contrôle de l'organisation (contrôle familial, financier, managérial, social) et la légitimité.

7 La théorie de la collusion s'est développée au début $\mathrm{du} \mathrm{xx}^{\mathrm{e}}$ siècle dans un contexte de crainte d'une constitution de cartels remettant en cause l'idéologie de la concurrence suite à la réflexion menée par le congrès américain qui s'est concrétisée par le vote du «Clayton Act» en 1914 interdisant tout partage d'administrateurs entre firmes concurrentes. Elle suppose une entente entre sociétés concurrentes : banques, sociétés industrielles... Cette politique, plus profitable qu'une situation de concurrence, se concrétise notamment par le partage d'administrateurs afin de contrôler conjointement les ressources rares, d'échanger des informations et de partager les marchés. La 
recherche académique n'a toutefois pas permis de conclure définitivement quant à la validité de cette perspective (Pennings, 1980 ; Carrington, 1981 ; Burt, 1983).

La théorie de la dépendance envers les ressources estime que la puissance d'une organisation dépend de sa capacité à accéder à des ressources financières, humaines, techniques, matérielles... qui lui sont vitales. Ne pouvant les produire à moindre coût, l'entreprise doit s'approvisionner à l'extérieur. Compte tenu des coûts et de l'aléa associés aux transactions sur le marché, la cooptation d'un représentant du fournisseur peut permettre de stabiliser la relation entre les partenaires en maîtrisant les sources d'incertitude de leur environnement (Allen 1974, Pfeffer et Salancik 1978, Burt 1983).

L'exploitation de l'activité de l'entreprise générant (normalement) une création de richesses, les divers requérants sont en compétition pour s'assurer le contrôle de l'organisation afin de s'attribuer une rémunération. La problématique du contrôle de l'organisation a donné naissance à plusieurs théories au gré de la transformation de la nature du capitalisme. À la fin du XIX siècle, quelques grandes familles détiennent des participations au capital leur conférant une position de contrôle dans les principales entreprises. La théorie du contrôle familial suppose que ces familles complètent le contrôle des sociétés appartenant à leur empire (en plus des liens financiers), par l'existence d'administrateurs communs. Les liens familiaux sont, dans cette perspective, l'un des moyens par lequel la bourgeoisie financière (française) assure et reproduit dans le temps son contrôle sur les capitaux qu'elle met en valeur (Bellon 1980).

La théorie du capital financier supporte l'idée d'une prééminence des grands établissements bancaires qui subordonnent le financement du secteur industriel et commercial, sous forme de prêts et de participations au capital, au détachement d'administrateurs. Le but est d'abord de protéger leurs intérêts en contrôlant les actions de la direction (Mintz et Schwartz 1981a, 1981b). Ces liens ne sont toutefois pas systématiques (Mizruchi et al. 1995) car les banques disposent d'un nombre limité de personnes qualifiées et peuvent recourir à d'autres moyens de protection (garanties).

11 L'émergence d'entreprises d'envergure nationale, l'expansion du gouvernement fédéral centralisé à partir des années 30 et l'apparition d'un complexe militaro-industriel au sortir de la seconde guerre mondiale aux U.S.A. ont remis en cause le pouvoir détenu par les banques au profit de grands propriétaires et des managers. Le capitalisme managérial est issu d'une dilution et d'une démocratisation de la propriété des grandes entreprises suite aux appels successifs aux marchés financiers, nécessaires pour faire face aux coûts croissants des investissements (passage à la production de masse...). Berle et Means (1932) dénoncent ainsi l'existence d'un groupe de dirigeants ayant, selon eux, accaparé le pouvoir dans les grandes entreprises. Il s'ensuit une séparation croissante entre la propriété et le contrôle des grandes sociétés avec un risque de divergence d'intérêts entre des managers plus rétifs à la prise de risque que ne le sont les actionnaires. La maximisation de la valeur des fonds propres pourrait ne pas être l'objectif principal d'agents désirant minimiser le risque supporté par leur capital humain et profiter de leur aptitude à prélever la rente organisationnelle. Emerge la théorie managériale qui énonce notamment que les liens humains entre conseils sont issus d'une volonté des dirigeants, relativement affranchis du contrôle des actionnaires et des prêteurs, de favoriser les relations qu'ils entretiennent avec les autres requérants d'importance (fournisseurs, clients, État). Pour ce faire, ils cooptent dans leur conseil d'administration des personnalités prestigieuses ou des individus supposés promouvoir ce type de relations. Par là-même, cela peut conduire à faciliter le contrôle des ressources comme cela a été vu 
précédemment. Mais cette indépendance relative de la direction peut aussi générer des dysfonctionnements.

12 À la fin du $\mathrm{xx}^{\mathrm{e}}$ siècle, suite à nombreux cas d'abus et de mauvaise gestion de la part d'équipes dirigeantes sans réels contre-pouvoirs, le capitalisme managérial laisse place au capitalisme institutionnel (Windolf 1999) où les investisseurs institutionnels reconcentrent la propriété des actions. Ils restaurent, ce faisant, le pouvoir conféré aux détenteurs d'actions qui avait été confisqué par les managers. Se développe alors aux USA la théorie de l'enracinement, extension de la théorie de l'agence, qui estime notamment que l'échange de mandats d'administrateurs entre dirigeants professionnels leur permet d'amoindrir la menace de sanction émanant des actionnaires, des prêteurs et du conseil d'administration (Maati 1999). Les réseaux de dirigeants, perçus négativement par la perspective disciplinaire, peuvent aussi apparaître comme un vecteur de mobilisation des compétences et comme des mécanismes permettant de mettre à l'épreuve les schémas cognitifs des dirigeants et de prévenir ou corriger leurs erreurs. Pour la finance comportementale et son prolongement qu'est la gouvernance cognitive, le conseil d'administration pourrait être composé de façon à créer des compétences, à faciliter l'apprentissage organisationnel et à réduire les coûts cognitifs en harmonisant les schémas cognitifs (Charreaux 2005).

La volonté de contrôler les organisations peut aussi être le fait d'un groupe d'individus. Selon la théorie de la cohésion de classe sociale, un groupe humain (dont les liens sont extra familiaux) peut maintenir sa domination politique et sociale à travers la cohésion issue $d u$ partage d'administrateurs au sein d'entreprises vues alors comme des instruments d'accumulation du capital (Radcliff 1980).

D'après la théorie néo-institutionnelle, les investisseurs prennent en considération les perspectives économiques de la société et la qualité de son management lorsqu'ils décident de s'impliquer dans une société. En cooptant des individus liés à des organisations de référence du point de vue économique, la firme signale aux investisseurs potentiels sa légitimité. Cette dernière facilite par là même le contrôle des ressources. Les réseaux sociaux sont alors vus, entre acteurs isolés et dispositifs institutionnels globalisés, comme étant à la fois un niveau d'analyse et un mécanisme de transformation d'un champ organisationnel (DiMaggio et Powell 1983, North 1990, Scott 2001).

Ces diverses théories sont-elles vérifiées dans la réalité et, si tel est le cas, ont-elles une influence significative sur l'architecture du réseau? Dans une perspective structurale, la théorie du Petit Monde (Milgram 1967, Watts 1999, Newman, Strogatz et Watts 2001) suppose l'existence d'une nature non planifiée du réseau des administrateurs qui est vu comme un système complexe pourvu notamment d'une résistance aux pressions extérieures. Pour Watts (1999), la transmission d'un élément (information, virus...) au sein d'une grande population est amplifiée grâce à quelques individus, des "chevilles ouvrières", dont les liens personnels favorisent la propagation et, ce faisant, transforment le "grand monde" en "petit monde". Grâce à eux, il ne faut que peu d'intermédiaires pour que deux individus sélectionnés au hasard puissent se rencontrer. Autrement dit, un monde est petit si les acteurs qui le constituent sont tous relativement proches les uns des autres malgré l'importance de la population. Milgram (1967) a mis en lumière cette architecture particulière en ayant révélé, grâce à sa célèbre expérience, les fameux "six degrés de séparation»: seuls six intermédiaires sont nécessaires, en moyenne, pour que deux personnes choisies aléatoirement dans la population mondiale puissent entrer en contact. Watts (1999) a formalisé le phénomène et a fourni une base à 
partir de laquelle se sont développées de nouvelles recherches sur l'architecture des réseaux sociaux. L'architecture Petit Monde est caractérisée notamment par la présence de grappes élémentaires conduisant à un regroupement élevé entre les acteurs. Toutefois, la moyenne des plus courtes distances entre les individus pris deux à deux demeure faible.

Pour Davis, Yoo et Baker (2002), les caractéristiques du réseau social des administrateurs américains sur une longue période valident la théorie du petit monde alternativement à la théorie du capital financier. L'article fondamental de Newman, Strogatz et Watts (2001) montre que l'architecture du réseau des administrateurs américains de "Fortune 1000 » en 1999 ne diffère pas de celle d'un réseau aléatoire équivalent en termes de distributions. La structure globale du réseau n'est donc pas influencée par les stratégies individuelles ou collectives pour autant qu'elles existent. Cela remet alors en cause la perspective négative qu'ont les opinions publiques quant aux réseaux d'administrateurs. En France, Kadushin (1995) et Morin (1996) appuient l'idée d'une dominance des banques aussi bien dans la dimension capitalistique que dans la dimension sociale. Sur une période plus récente, Chabi et Maati (2006) corroborent la thèse du petit monde en observant le réseau des administrateurs français des sociétés du CAC 40 de 1996 à 2004. Maati (2008) parvient à la même conclusion à l'échelle de l'Europe.

\section{Méthodologie d'analyse du réseau social}

Il s'agit maintenant de présenter le réseau social en justifiant son statut en tant qu'objet d'analyse, puis de spécifier les liens sociaux le caractérisant ainsi que les variables permettant de distinguer des sous-réseaux.

\subsection{Le réseau social}

Le réseau social étudié est celui des membres des conseils d'administration des compagnies d'assurance en France en 1881. Il s'agit, d'une part, de mettre en oeuvre une méthodologie permettant d'améliorer les modèles visant à comprendre le processus de création et de captation de la rente organisationnelle par les acteurs, dans une perspective disciplinaire ou cognitive de la gouvernance. Ce travail est, vu sous ce prisme d'analyse, la première phase d'un travail plus large mêlant les concepts tirés de la théorie des graphes à d'autres, plus traditionnels. D'autre part, dans une perspective historique, il est possible de se questionner sur l'existence d'une structure en réseau dans un secteur d'activité en croissance et dont la création est relativement récente. Si tel est le cas, quel type d'architecture domine?

Le choix du secteur de l'assurance découle de ses spécificités relativement au capitalisme français. En effet, son histoire est ancienne puisqu'elle remonte à l'Ancien Régime avec le développement initial des assurances maritimes. Elle se poursuit à la fin de l'Ancien Régime avec la naissance d'autres branches qui ne se développeront réellement qu'à la Restauration, la Révolution ayant significativement entravé leur expansion. L'assurance constitue aussi un domaine où la confiance est essentielle, car les compagnies d'assurance ont plus besoin de crédit que d'argent puisque, bien gérées, leurs frais sont faibles d'une part, et les primes sont encaissées avant le règlement des sinistres d'autre part. Cette particularité explique que les compagnies d'assurance ont des caractéristiques uniques au $\mathrm{XIX}^{\mathrm{e}}$ siècle. D'abord leurs actions ont un nominal très élevé : habituellement 5000 francs 
contre 12500 francs pour la Générale-maritime lorsque le salaire d'un journalier est de 2 francs. Après la guerre de 1870, le prix des actions de ce secteur chute. Leur capital est très faiblement libéré, les compagnies n'appelant habituellement qu'un cinquième de leur capital, celui-ci n'étant pas toujours versé en espèces. Il peut consister en un dépôt de fonds publics dont les arrérages appartiennent à l'actionnaire. En cas de besoin, le conseil d'administration dispose du dépôt de garantie et peut appeler (de façon rarissime) le capital non libéré. En contrepartie, les actions sont nominatives et la vente d'une action peut être conditionnée à l'acceptation du nouvel actionnaire par le conseil d'administration afin de n'admettre que les acquéreurs jouissant d'un bon crédit. Cette quasi cooptation des nouveaux actionnaires permet aussi au conseil d'administration de protéger la répartition du pouvoir. Très souvent, il faut avoir possédé pendant un certain temps un nombre minimum d'actions pour participer à l'assemblée générale des actionnaires où le droit de vote est lui-même contingenté. Le mode de cotation des actions des compagnies d'assurance est spécifique car elles sont évaluées en pourcentage de bénéfices ou de perte par rapport à leur valeur nominale.

Outre leurs compétences, les administrateurs des compagnies d'assurance se doivent de posséder un nom, une particule ${ }^{1}$, un titre, des décorations tels que leur énoncé inspire confiance auprès des épargnants et serve de garantie pour les opérations de la compagnie. C'est ainsi que l'on trouve les grands noms de l'ancien régime (comte de Lévis-Mirepoix, comte Rozan, comte de Clermont-Tonerre, comte de La Rochefoucauld, etc.). Les compétences peuvent être techniques. Par exemple, A. de Courcy, spécialiste reconnu de l'assurance et auteur de nombreux ouvrages et articles, est Directeur de la Compagnie d'assurance Générales Maritimes (depuis 1837) et membre du conseil d'administration des branches maritime, incendie et vie des Assurances générales, Thomas de Colmar, inventeur de l'arithmomètre, est le fondateur du Soleil. Plusieurs banquiers sont aussi administrateurs de compagnies (Pillet-Will, Hentsch, Marcuard, Mallet, Odier, de Waru,...) et certains combinent deux qualités comme le vicomte de La Panouse. D'autres viennent plus récemment d'être anoblis, tels les Mallet, Hottinguer, Rothschild, etc. Dans d'autres cas, il s'agit simplement de grands industriels comme Dollfuss ou Koechlin. Les titres ("général », " ancien député », etc.) et les décorations (légion d'honneur) participent aussi à cette mise en confiance,

21 Les actes des administrateurs et des dirigeants se traduisent dans les comptes qui sont examinés non seulement lors de l'Assemblée générale, mais aussi par certains administrateurs spécialement délégués à cette fonction. Ceux-ci sont appelés membres $d u$ conseil de surveillance ou censeurs et ont le droit de se faire ouvrir les comptes et la caisse, de se faire communiquer les registres ainsi que de voir les correspondances. Le rôle du censeur est particulièrement délicat car il lui est possible d'agir à l'encontre de la direction. La présence, au sein de Nationale-incendie, de censeurs tels le banquier Odier et le comte de Pillet-Will inspire confiance.

22 La structure spécifique de l'actionnariat, issue de la nécessité de pouvoir mobiliser rapidement de fortes sommes, et le rôle majeur joué par le conseil d'administration pour inspirer la confiance expliquent l'intérêt du secteur de l'assurance à la fin du XIX siècle en tant qu'objet d'analyse de cette étude. Évidemment, le secteur d'activité de l'assurance demeure inséré dans le capitalisme français, celui-ci l'étant lui-même dans le capitalisme mondial. Même si l'assurance est un secteur spécifique avec des règles et des procédures particulières, certains grands capitalistes qui appartiennent au réseau social sont aussi d'éminents capitalistes dans le reste de l'industrie ou de la banque. Il en est ainsi de tous 
les banquiers d'affaire (Rothschild, Hentsch, Odier...) ou de leurs commensaux (Denormandie, Bartholoni). D'autres individus apparaissent parmi les membres des conseils d'administration des Compagnies d'assurances, mais aussi dans d'autres secteurs d'activité. L'étude sur un secteur particulier peut être perçue comme partielle, à l'instar de toute analyse de sous-ensemble. Cette particularité est toutefois limitée par les spécificités sectorielles très fortes.

L'information provient des relevés d'Alphonse Courtois qui mentionnent, pour chaque compagnie, sa raison sociale, la branche de risque couverte (déterminée par le nom de la compagnie ou par son objet), son année de création et le nom des administrateurs de chaque compagnie cotée, avec leurs titres éventuels. De plus sont indiquées diverses fonctions exercées dans la compagnie, la direction et le contrôle notamment. Alphonse Courtois publie en 1856 la première édition de son ouvrage Des opérations de Bourse qui porte pour sous-titre Manuel des fonds publics et des sociétés par actions et compte 536 pages. Plus tard, les deux parties seront séparées en deux ouvrages dont la première discutera des opérations de bourse et de change en défendant les opérations "d'agiotage » (de spéculation dirait-on aujourd'hui) qui sont encore sous le coup de la loi et du tribunal à l'époque, alors même que ces opérations se pratiquaient à grande échelle. Le Manuel prendra son autonomie et connaîtra de nombreuses éditions, progressivement enrichies, pour deux raisons. D'une part, le capitalisme français s'étoffe au cours du XIX ${ }^{e}$ siècle. Les investissements se multiplient et de nouveaux secteurs s'ouvrent au financement boursier. Par exemple, en 1819, seules deux actions (Banque de France et Vieux Ponts de la Seine) sont traitées par la bourse de Paris en dehors du secteur de l'assurance qui vient de se lancer et dont 7 actions sont cotées. A contrario, en 1881, la cote contient 15 secteurs d'activité, déclinés en multiples sous-secteurs et en milliers d'entreprises. D'autre part, Alphonse Courtois est nommé à la tête du Service d'Etudes Financières (SEF) du Crédit Lyonnais en 1871, ce qui lui permet d'accéder à des sources d'informations étoffées et conséquentes. L'édition de référence, "le Courtois», est celle de 1883, la huitième du Manuel des fonds publics et des sociétés par actions qui compte 1283 pages et dont les relevés s'arrêtent en 1881. C'est à partir de cette dernière qu'a été constituée la base de données des liens sociaux. Traité en 83 pages sans compter les compagnies étrangères sur 50 pages, le secteur des assurances est l'un des plus importants après les chemins de fer (90 pages) alors que les banques françaises hors Banque de France n'occupent que 65 pages (50 pages supplémentaires pour les banques étrangères). L'industrie métallurgique n'est traitée qu'en 32 pages.

\subsection{Les liens sociaux et sous-réseaux}

24 Les liens sociaux sont mesurés à partir du portefeuille de mandats détenu par les administrateurs des sociétés du gisement précité dans une perspective bi-dimensionnelle sociétés-conseils d'administration/individus-administrateurs. Ces acteurs sont ceux qui dirigent la compagnie, tant dans l'orientation générale (le conseil d'administration avec à sa tête un président aidé d'un éventuel secrétaire-général), que dans la gestion quotidienne (le directeur, le directeur-adjoint éventuel) et l'organe de contrôle (les commissaires, les censeurs, etc.).

Il est possible, à partir de ce réseau global, de spécifier des sous-réseaux afin d'affiner la perspective. Il en est ainsi du sous-ensemble des administrateurs portant un nom à particule et celui des administrateurs portant un titre. En effet, il peut être avantageux de 
coopter au sein du conseil d'administration des personnes dotées de noms à particule, de titres nobiliaires, de prestige et de décorations, même si la monarchie a été abolie, afin d'inspirer confiance à l'épargnant. Les particules «de», «du », «d'« telles qu'elles sont indiquées dans l'édition de Courtois de 1883 ont donc été relevées même si les particules font, parfois à tort, irrésistiblement penser à la noblesse, gage d'une expérience de gestion millénaire dans l'imaginaire collectif. Il faut noter que les grands banquiers, qui viennent d'acheter des titres (Rothschild, ...), n'apparaissent jamais avec leur particule ou leur titre (baron...).

Les individus dotés de titres nobiliaires tels que comte, duc, prince, etc., docteur (cité à plusieurs reprises) et général (malgré la récente défaite, la population française semble toujours admirer son armée) représentent un autre sous-réseau.

Enfin, il est possible de distinguer, parmi les administrateurs, les contrôleurs (qui prennent différents noms à l'époque tels que commissaire, censeur, etc.) des administrateurs cumulant (directeur, directeur adjoint, secrétaire-général, etc.) ou non une fonction de direction dans la compagnie d'assurance.

Dans la dimension sociétés, les compagnies peuvent être regroupées selon le risque assuré: la vie, l'incendie, le risque maritime, la grêle, l'assurance coloniale, la coassurance, la réassurance et l'accident.

\section{Statistiques préliminaires}

Le gisement étudié comporte, dans la dimension sociétés, 95 observations. Dans la dimension individus, la population incorpore 660 individus disposant de 1080 mandats. La différence entre le nombre d'individus et le nombre de postes naît de l'existence d'administrateurs multi-mandats qui permettent aux entreprises d'être mutuellement connectées.

La répartition des mandats entre les individus est très asymétrique : 390 administrateurs mono-mandat $(59,09 \%$ du total des individus) côtoient 270 administrateurs multimandats ( $40,91 \%$ du total des individus). Ces derniers, qui seuls permettent l'émergence d'un réseau social en connectant les conseils entre eux, détiennent ensemble $63,9 \%$ des mandats. Les 124 administrateurs ayant 3 mandats ou plus et comptant pour $18,8 \%$ des administrateurs, détiennent ensemble $36,9 \%$ des mandats. À l'extrémité de la distribution, deux administrateurs détiennent 5 mandats $(0,30 \%$ des individus, $0,9 \%$ des mandats).

31 En moyenne, il existe 1,67 administrateur portant un titre par compagnie (représentant $12,28 \%$ du conseil d'administration) alors qu'il existe 2,8 administrateurs qui portent une particule par compagnie (soit $20,6 \%$ des membres d'un conseil d'administration). Les contrôleurs comptent pour 3,45 individus en moyenne par compagnie et représente $25,38 \%$ du conseil d'administration.

32 Dans la dimension sociétés, le gisement incorpore trois risques bien déterminés : la vie, l'incendie et le risque maritime. Les autres risques (grêle, assurance coloniale, coassurance, réassurance) ne sont représentés que par une ou deux compagnies et sont regroupés dans une catégorie "Autres risques ». Cette dernière incorpore aussi le risque Accident qui, couvert par une seule compagnie avant la guerre de 1870, voit émerger 11 nouvelles compagnies entre 1871 et 1881. 

créée des centaines d'années avant les autres types d'assurances, l'assurance maritime est relativement simple à mettre en œuvre (l'arrivée d'un bateau se constate aisément), suscite moins de tentatives de fraudes (comparativement à l'assurance incendie) et ne pose guère de problèmes moraux (relativement à l'assurance vie). Son activité ne cesse pas durant la période de la Révolution et de l'Empire au contraire des autres branches de risques. Enfin, il s'agit d'une activité mise en œuvre par des professionnels du transport maritime, à titre d'activité annexe, et non d'une branche d'activité à bâtir ex nihilo. Comme les professionnels du transport maritime demeurent en partie dans les ports, il n'est pas surprenant de compter à la fois plus de bourgeois de province et moins de descendants d'aristocrates à titre ou à particule. En même temps, à mesure de l'avancement du siècle, cette branche d'activité va tendre progressivement vers les autres, les nouvelles compagnies d'assurance maritime se distinguant moins des autres créées au même moment. Enfin, l'évaluation financière de l'activité d'assurance maritime est la branche de risque qui pose le plus de difficultés car, si elle distribue un taux de dividende élevé, ses titres se valorisent très peu au contraire des autres branches de risques, peut-être parce que le risque maritime est systématiquement mal évalué et qu'une guerre des prix ravage le secteur du transport et de l'assurance maritime. En effet, le milieu du XIXe siècle voit le crépuscule de la marine à voile et la montée en puissance de la marine à vapeur, la fin de la marine en bois et la généralisation de la construction en fer. Or cette double révolution technique est à la fois mal vécue économiquement et techniquement par surévaluation systématique de l'innovation, par exemple quand il apparaît que les bateaux en fer sont plus fragiles qu'il n'y paraît dans les tempêtes.

Il s'agit maintenant de déterminer dans quelle mesure la structure du réseau des administrateurs des principales compagnies d'assurance sur le marché français à la fin du XIX ${ }^{e}$ siècle peut-elle être expliquée par les stratégies de certains acteurs qui viseraient à protéger leurs intérêts. Si le réseau réel ne peut se distinguer significativement d'un réseau issu d'un processus aléatoire d'allocation des liens, cela signifie que même si des stratégies locales peuvent être engagées, elles n'ont pas de conséquences sur la structure globale du réseau.

\section{Le réseau des administrateurs est-il un petit monde?}

Tout réseau d'administrateurs, composé de $N$ individus siégeant au total dans $M$ conseils, observé à une date $t$ peut être représenté par une matrice d'affiliation binaire ayant $N$ lignes et $M$ colonnes. Toute présence d'un administrateur Mi dans un conseil $N i$ est codé par une valeur égale à l'unité, le solde des valeurs de la matrice étant égal à zéro.

Économie et institutions, 18-19 | 2012 

premiers voisins). Il existe alors parmi ses premiers voisins au plus $z_{\mathrm{i}} \times\left(z_{\mathrm{i}}-1\right) / 2$ liens (lorsque le voisinage est complètement connecté). $C_{i}$ mesure la part des liens observables dans le voisinage de i. C est la moyenne des $C_{i}$ sur tous les individus de la population. $\mathrm{C}$ donne donc une mesure de la probabilité que deux amis d'un individu soient également amis.

Distance géodésique moyenne : la distance géodésique entre deux acteurs i et h est la plus courte distance les séparant: $\mathrm{L}_{\mathrm{ih}}$. La distance géodésique moyenne d'un individu i, notée $\mathrm{L}_{\mathrm{i}}$, est la somme des distances géodésiques liant $\mathrm{i}$ à tous les autres membres de son réseau, le tout rapporté au nombre d'acteurs liés à i. La distance géodésique moyenne du réseau, L, correspond à la moyenne des distances géodésiques individuelles de tous les acteurs du réseau.

43 Graphe de Caveman connecté : un graphe complètement connecté possède un coefficient de clustering égal à 1 . Le graphe de Caveman est constitué par un ensemble de $\mathrm{N}=(z+1)$ cliques $^{3}$ à l'intérieur desquelles tous les éléments sont liés les uns aux autres. De manière à lier toutes les cliques, un lien est enlevé de chacune et est ensuite utilisé pour connecter une clique donnée à sa voisine pour aboutir au graphe de Caveman connecté.

Graphe aléatoire (figure 1) : les observations sont liées par des connexions distribuées au hasard pour un nombre d'acteurs et de liens donnés. 
Figure 1 : Les architectures types de réseau
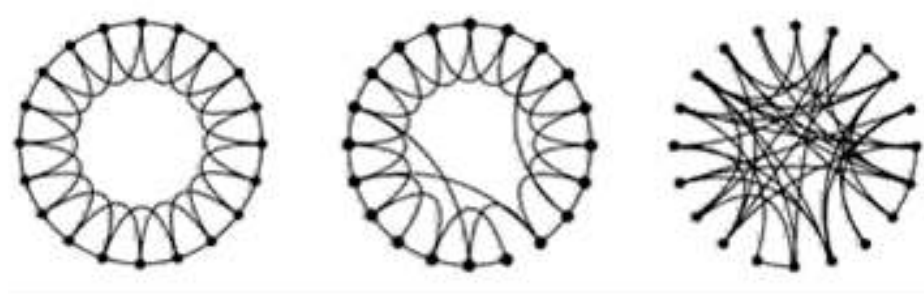

(1) Monde régulier

(2) Petit Monde

(3) Monde aléatoire

Source : Watts (1999)

\subsection{Conditions préalables}

Selon Watts (1999), les conditions préalables sont :

1. Le réseau est grand: $\mathrm{N} \gg 1$, condition affirmant qu'il ne serait pas étonnant que tous les membres d'un graphe de petite taille se connaissent.

2. Le réseau est clairsemé : $\mathrm{z} \ll \mathrm{N}$. En moyenne, les individus ne sont liés qu'à $\mathrm{z}$ autres individus. Chacun ne dispose donc que de peu de relations directes relativement à la taille de la population.

3. Le réseau est décentralisé : $\mathrm{z}_{\max } \ll \mathrm{N}$, avec $\mathrm{z}_{\max }$ le nombre de liens qu'un individu peut nouer au maximum. Il n'y a donc pas d'acteur dominant auquel la plupart des autres sont liés. Dans le cas contraire, cela permettrait aux autres membres du réseau d'entrer en contact mutuellement et réduirait les distances.

4. Le réseau est formé de cercles d'acteurs : le coefficient de clustering $C$ est élevé.

\subsection{Les réseaux petit monde}

Outre les propriétés citées précédemment, un graphe "petit monde» (figure 2) est caractérisé par une faible distance géodésique moyenne L. C'est une forme intermédiaire aux deux graphes extrêmes que sont le graphe régulier de type graphe de Caveman connecté et le graphe aléatoire (figure 2). C'est un graphe régulier, où chaque élément est lié à ses $\mathrm{z}_{\mathrm{i}}=\mathrm{z}$ voisins directs, auquel il suffit d'ajouter une dose d'aléa qui rend le monde petit. Celle-ci découle de l'existence de liens particuliers dans le réseau, des « raccourcis » (ou "shortcuts"), qui, lorsqu'ils sont supprimés, accroissent la distance entre les deux acteurs qu'ils connectent à une valeur strictement supérieure à 2. Pour Watts et Strogatz (1998), il suffit d'un très faible pourcentage de "raccourcis » aléatoires pour rendre le monde petit. Les raccourcis du réseau social étudié proviennent des administrateurs multiples qui seront étudiés plus précisément par la suite.

Pour pouvoir caractériser les valeurs de $\mathrm{C}$ et $\mathrm{L}$ observées, il est nécessaire de s'adosser à des graphes connus. La référence pour les réseaux ayant un coefficient de clustering élevé est le graphe de Caveman connecté. Pour ce graphe, selon Watts (1999), 


$$
\mathrm{C}_{\text {caveman }} \approx 1-\frac{6}{\mathrm{z}^{2}-1} \text { et } \mathrm{L}_{\text {caveman }} \approx \frac{\mathrm{N}}{2(\mathrm{z}+1)}
$$

La référence pour les réseaux avec une moyenne $L$ faible est le graphe aléatoire pour lequel :

$$
\mathrm{C}_{\text {random }}=\frac{\mathrm{Z}}{\mathrm{N}} \text { et } \mathrm{L}_{\text {random }}=\frac{\ln (\mathrm{N})}{\ln (\mathrm{Z})}
$$
indirectement. Par définition, deux individus sont membres de la même composante s'il existe un chemin entre eux. La composante n'est pas pour autant nécessairement un graphe complet puisque les chemins liés entre les acteurs pris deux à deux peuvent avoir une longueur strictement supérieure à l'unité. 
Tableau 1.1 : La distribution des composantes d'administrateurs

\begin{tabular}{|c|c|c|}
\hline Nombre de composantes & Nombre d'individus & Nombre de sociétés \\
\hline 1 & 466 & 69 \\
\hline 1 & 29 & 4 \\
\hline 1 & 27 & 4 \\
\hline 1 & 26 & 2 \\
\hline 1 & 16 & 4 \\
\hline 1 & 15 & 1 \\
\hline 1 & 14 & 1 \\
\hline 1 & 13 & 1 \\
\hline 2 & 12 & 1,2 \\
\hline 1 & 11 & 3 \\
\hline 1 & 8 & 1 \\
\hline 1 & 6 & 1 \\
\hline 1 & 5 & 1 \\
\hline 14 & 660 & 95 \\
\hline
\end{tabular}

Tableau 1.2: La distribution des composantes de conseils d'administration

\begin{tabular}{|l|l|l|}
\hline Nombre de composantes & Nombre de sociétés & Nombre d'individus \\
\hline 1 & 69 & 466 \\
\hline 3 & 4 & $16,27,29$ \\
\hline 1 & 3 & 11 \\
\hline 2 & 2 & 12,26 \\
\hline 7 & 1 & $5,6,8,12,13,14,15$ \\
\hline $\mathbf{1 4}$ & 95 & $\mathbf{6 6 0}$ \\
\hline
\end{tabular}

54 Les tableaux 1.1 et 1.2 montrent que les individus (donc les conseils) ne sont pas tous liés, même indirectement. Il existe une composante dominante formée de 466 administrateurs 
(70,60 \% des individus) correspondant à 69 conseils (72,63\% des conseils). S'ajoutent à ce groupe social 3 composantes regroupant 4 conseils chacune pour un nombre d'administrateurs allant de 16 à 29 , une composante formée de 3 conseils et 11 individus, deux composantes groupant 2 conseils (comprenant respectivement 12 et 26 administrateurs) et 7 conseils ne partageant aucun administrateur avec un autre conseil et dont la taille varie entre 5 et 15 administrateurs.

La figure 2 présente les différentes composantes d'acteurs dans la dimension sociétés (le nombre de liens et d'acteurs dans la dimension individus ne permet pas de représentation graphique claire).

Figure 2 : Les composantes de compagnies d'assurances

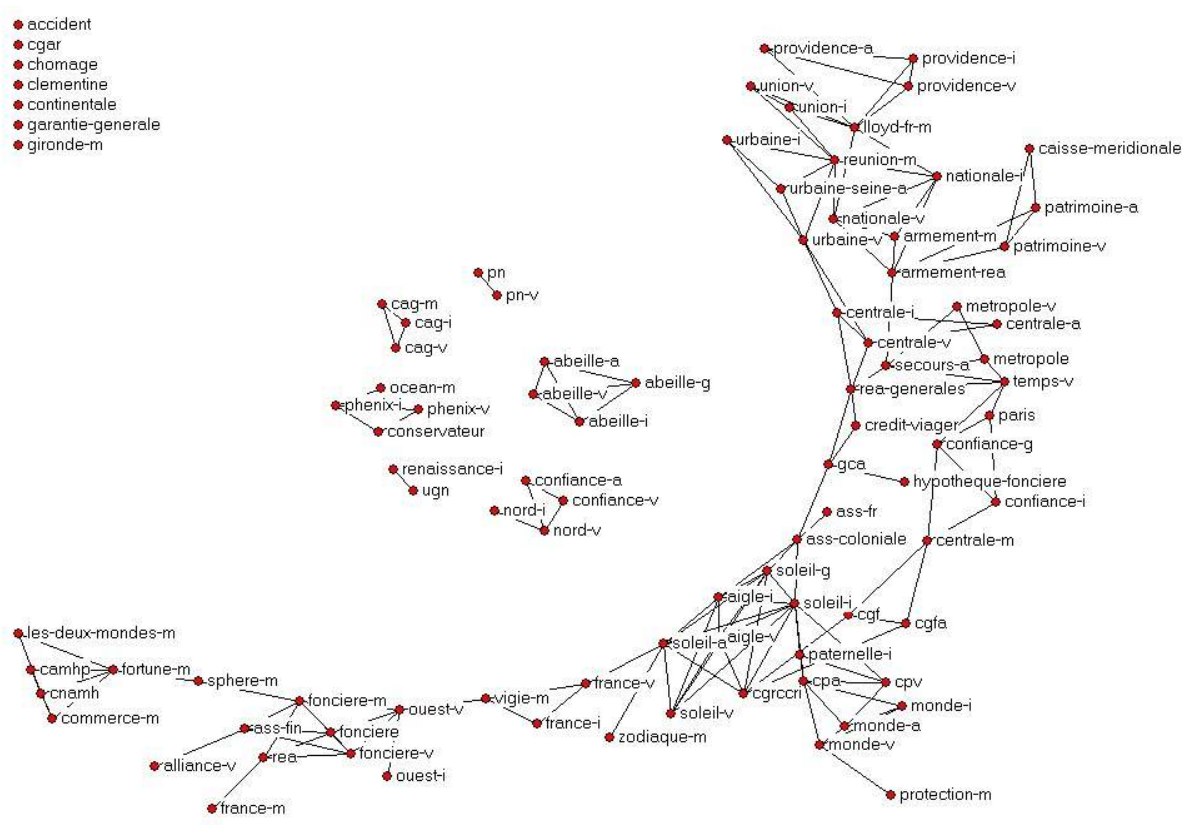

Il est possible d'observer les regroupements des compagnies par branche de risques depuis qu'une décision du Conseil d'État de 1826 oblige les compagnies à scinder les différentes branches, ce qui revient à créer des groupes. Il existe ainsi des groupements de deux, trois, voire quatre types de risques assurés par un groupe de compagnies. Cela est particulièrement manifeste dans les îlots centraux en ce qui concerne l'Abeille ou la Compagnie des Assurances Générales, mais se retrouve aussi dans la composante géante. Dans cette dernière, certaines proximités sont marquantes: les groupes Aigle et Soleil fondés par un père afin d'établir deux fils; d'autres sont plus inattendues : la Compagnie des Assurances Nationales avec l'Union ou l'Urbaine. Enfin, il existe un ensemble de Compagnies isolées qui ne présentent aucune spécificité particulière (branche de risque, date de création...).

\subsubsection{Les voisinages directs}

57 Il s'agit à présent de calculer le nombre moyen d'administrateurs avec lesquels un administrateur est en contact direct à travers les liens noués dans les conseils d'administration. La centralité de degré mesure le volume d'activité d'un individu. Elle représente le nombre de premiers voisins d'un administrateur indépendamment du nombre de conseils où se croisent les individus. 
Le tableau 2 montre que l'administrateur disposant du voisinage direct le plus grand côtoie 40 personnes dans les conseils où il siège. Sont présents deux individus à particule qui possèdent par ailleurs des titres, les comtes de Rozan et de Rancy.

Tableau 2 : Les administrateurs ayant le plus grand voisinage direct

\begin{tabular}{|l|l|}
\hline Identité & $z_{i}$ \\
\hline Dormoy & 40 \\
Brullé & 37 \\
de Rozan & 35 \\
Buchot & 34 \\
Blondel & 33 \\
Binder & 31 \\
de Rancy & 31 \\
Guépratte & 29 \\
Mazerat & 29 \\
Pépin-Lehalleur & 29 \\
\hline $\mathbf{z}^{(1)}$ & $\mathbf{1 3 , 8 4}$ \\
\hline
\end{tabular}

(1) Valeur mesurée au sein de la composante la plus grande.

\subsubsection{Les distances}

59 En suivant la méthodologie de Watts, la distance géodésique moyenne réelle $\mathrm{L}_{\text {observé }}$ va être comparée à celle découlant d'une distribution aléatoire des liens sociaux $\mathrm{L}_{\text {random}}$. Le tableau 3 montre les administrateurs qui sont, en moyenne, séparés de tous les autres par les distances les plus faibles. La distance moyenne est égale à 7,07. Au sein de la composante la plus importante, il faut donc 6 intermédiaires en moyenne pour que deux administrateurs pris au hasard puissent communiquer, résultat proche de celui de Milgram pour une société ouverte.

Tableau 3 : Les administrateurs de la composante majeure les plus proches des autres

\begin{tabular}{|l|l|}
\hline Identité & $\boldsymbol{L}_{\boldsymbol{i}}$ \\
\hline de Ham & 4,83 \\
\hline de Tracy & 4,858 \\
\hline Plouviez & 4,927 \\
\hline Demachy & 4,976 \\
\hline Sapial de Lencia & 5,019 \\
\hline Martineau des Chesnez & 5,105 \\
\hline
\end{tabular}




\begin{tabular}{|l|l|}
\hline de Mornay & 5,114 \\
\hline Gosselin & 5,168 \\
\hline Vengohechea & 5,222 \\
\hline Leclert & 5,269 \\
\hline Moyenne $^{(1)}$ & $\mathbf{7 , 0 7 9}$ \\
\hline Minimum $^{(\mathbf{1})}$ & $\mathbf{1}$ \\
\hline Maximum $^{(\mathbf{1})}$ & $\mathbf{1 7}$ \\
\hline
\end{tabular}

(1) Valeur mesurée au sein de la composante la plus grande.

60 Le graphe des assureurs est aussi caractérisé par la totale dissociation entre les administrateurs ayant le plus grand voisinage direct et les administrateurs les plus proches des autres. En effet, aucune des personnes référencées dans le palmarès précédent n'est présente dans le tableau 3. Sont aussi présents cinq individus à particule et quatre possédant des titres, soit une proportion substantiellement plus élevée que celle du conseil d'administration moyen.

\subsubsection{Les coefficients de clustering}

61 Seuls les administrateurs ayant des mandats multiples ont un coefficient de clustering inférieur à un puisque le réseau composé des administrateurs d'un seul et même conseil d'administration est un réseau complet, chaque administrateur étant directement liée aux autres. Les administrateurs mono-mandat ont alors nécessairement un coefficient de clustering égal à l'unité. Les individus qu'ils rencontrent se trouvent dans le même conseil d'administration et par conséquent se connaissent également. Le tableau 4 met en évidence les administrateurs qui ont le plus de contacts avec des personnes qui ne se connaissent pas entre elles.

62 Le classement des administrateurs selon la valeur de leur coefficient de clustering fait apparaître de nouvelles individualités. Pour autant, il n'y a pas une totale dissociation entre les individus les plus proches des autres et ceux ayant le plus de contacts avec des personnes qui ne se connaissent pas entre elles.

Tableau 4 : Les administrateurs ayant le plus de contacts avec des personnes qui ne se connaissent pas entre elles

\begin{tabular}{|l|l|}
\hline Identité & $\boldsymbol{c}_{\boldsymbol{i}}$ \\
\hline Kolb-Bernard & 0,372 \\
\hline de Ham & 0,396 \\
\hline Malpas-Duché & 0,41 \\
\hline Lepoutre-Pollet & 0,413 \\
\hline
\end{tabular}




\begin{tabular}{|l|l|}
\hline Pernolet & 0,441 \\
\hline Monzie-Lasserre & 0,451 \\
\hline Péligot & 0,458 \\
\hline Plouviez Père & 0,462 \\
\hline Martineau des Chesnez & 0,481 \\
\hline de Ferrabouc & 0,488 \\
\hline$C^{(1)}$ & $\mathbf{0 , 9 0 4}$ \\
\hline
\end{tabular}

(1) Valeur mesurée au sein de la composante la plus grande.

63 Ce tableau montre la présence de trois individus à particule et de deux qui possèdent des titres, soit une proportion bien plus élevée que celle du conseil d'administration moyen. Trois administrateurs sont présents dans ce classement comme dans le précédent et aucun n'apparaît simultanément dans les classements relatifs au voisinage direct et au clustering.

64 La comparaison des trois tableaux (administrateurs ayant le plus grand voisinage direct, administrateurs de la composante majeure les plus proches des autres et administrateurs ayant le plus de contacts avec des personnes qui ne se connaissent pas entre elles) fait apparaître 27 administrateurs personnes physiques sur trente noms possibles. Seuls trois noms apparaissent deux fois : Plouviez, de Ham et Martineau des Chenez. Ceci ne semble guère suggérer la présence d'un nombre important de personnes occupant une position stratégique, soit pour mettre en contact des administrateurs qui se connaissent, soit en servant de lien à des administrateurs qui ne se connaissent pas.

\subsubsection{La validation de la structure « petit monde »}

Le tableau 5 récapitule les diverses statistiques nécessaires à la validation de la structure de type petit monde au sein du réseau des administrateurs.

Tableau 5 : Les indicateurs du petit monde

\begin{tabular}{|l|l|l|l|l|l|l|l|l|}
\hline $\boldsymbol{N}$ & $\boldsymbol{N}^{\prime}$ & $\boldsymbol{Z}$ & $\boldsymbol{Z}^{\prime}$ & $\boldsymbol{L}^{\prime}$ & $\boldsymbol{C}^{\prime}$ & $\boldsymbol{L}_{\text {random }}$ & $\boldsymbol{C}_{\text {random }}$ & quotient $S W$ \\
\hline 660 & 466 & 3,30 & 3,84 & 7,10 &, 9 & 2,3 & 0,029 & 10,04 \\
\hline
\end{tabular}

$N$ : nombre total d'observations,

$N^{\prime}$ : Nombre d'observations dans la plus grande composante,

$z$ : centralité de degré moyenne,

$z^{\prime}$ : centralité de degré moyenne dans la plus grande composante,

$L_{\text {random }}^{\prime}$ : distance géodésique moyenne dans un réseau aléatoire de même taille, approximée par $\ln \left(N^{\prime}\right) /$ $\ln \left(z^{\prime}\right)$,

$C_{r a n d o m}^{\prime}$ : coefficient de clustering moyen dans un réseau aléatoire de même taille approximé par $\mathrm{z} / \mathrm{N}$. 

probabilité que $\mathrm{A}$ et $\mathrm{B}$ se connaissent, sachant que $\mathrm{C}$ connaît $\mathrm{A}$ et $\mathrm{B}$. Elle est définie comme : $C_{\Delta}=3 \mathrm{x}$ nombre de triangles dans le graphe / nombre de trios d'administrateurs connectés. Le facteur 3 s'explique par le fait que chaque triangle contribue à trois trios d'administrateurs connectés, un pour chacun des trois administrateurs. $C_{\Delta}$ représente donc la probabilité que deux administrateurs soient liés sachant qu'ils côtoient tous deux un même autre administrateur. Les deux définitions sont relativement proches, elles diffèrent dans le sens des opérations effectuées. La première prend la moyenne des ratios alors que la seconde calcule le ratio des moyennes. est une définie par :

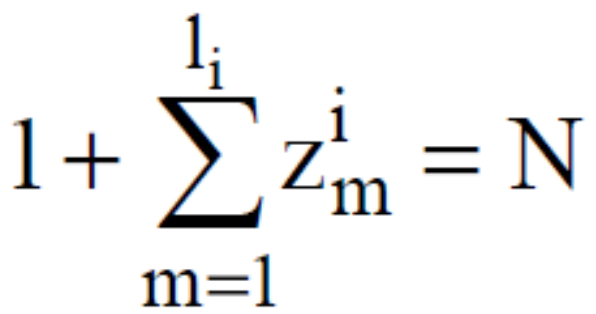


avec

\section{$\mathrm{z}_{\mathrm{m}}^{\mathrm{i}}$}

$\mathrm{m}=1, \ldots, \mathrm{l}_{\mathrm{i}}$

le nombre de $\mathrm{m}^{\text {ième }}$ voisins (remarque $: \mathrm{z}_{1}=\mathrm{z}$ );

$\mathrm{l}=\Sigma_{\mathrm{i}} \mathrm{l}_{\mathrm{i}} / \mathrm{N}$.

\subsection{Analyse du graphe des administrateurs}

La méthodologie, proposée par Newman et al. (2001), conduit à calculer les indicateurs du petit monde en se fondant sur les fonctions génératrices des moments des distributions de probabilité pour déterminer les valeurs théoriques de $C_{\Delta} l$ et $z_{1}$ dans un graphe aléatoire de même taille qui respecte la structure duale de nos données et qui possède exactement les mêmes distributions de taille de conseil et de nombre de mandats, tout le reste étant aléatoire. Les résultats figurent dans le tableau 6.

La fonction génératrice des moments de la distribution empirique du nombre de mandats pour la population des administrateurs des compagnies d'assurances est :

$$
f_{K}(x)=\frac{390 x+146 x^{2}+100 x^{3}+22 x^{4}+2 x^{5}}{660}
$$

et celle de la taille du conseil est donnée par :

$$
\mathrm{g}_{\mathrm{T}}(\mathrm{x})=\begin{aligned}
& 4 x^{5}+9 x^{6}+5 x^{7}+4 x^{8}+8 x^{9}+9 x^{10}+ \\
& 5 x^{11}+12 x^{12}+9 x^{13}+12 x^{14}+7 x^{15}+ \\
& 2 x^{16}+6 x^{17}+3 x^{18}
\end{aligned}
$$

\begin{tabular}{|c|c|c|c|c|}
\hline Type de graphe & $N$ & $c_{\Delta}$ & $L, l$ & $z$ \\
\hline Graphe observé( ${ }^{(1)}$ : & \multirow{3}{*}{660} & 0,82 & 3,54 & 13,30 \\
\hline Graphe aléatoire biparti: distribution de Poisson ${ }^{(2)}$ : & & 0,38 & 2,20 & 18,60 \\
\hline Graphe aléatoire biparti ${ }^{(3)}$ : & & 0,47 & 2,37 & 18,77 \\
\hline
\end{tabular}

Table 6 : Valeurs théoriques et observées des paramètres des graphes

$N$ : nombre total d'observations

$C_{\Delta}=3 \times$ nombre de triangles dans le graphe / nombre de trios d'administrateurs connectés

L: distance géodésique moyenne.

Longueur du chemin caractéristique $I=\left[\right.$ [ $_{j} I_{i} / N$.

z: centralité de degré moyenne.

(1) Graphe réel.

(2) Graphe aléatoire biparti construit à partir des fonctions génératrices des distributions de Poisson

du nombre de mandats et de la taille des conseils.

(3) Graphe aléatoire biparti construit à partir des deux fonctions génératrices des distributions réelles. 
Le nombre moyen de mandats par administrateur est égal à $\mu=1,63$ et chaque conseil dispose de $v=11,37$ administrateurs. Le clustering poissonien du graphe est largement inférieur au clustering réel, ce qui traduit le fait que les administrateurs tendent à se regrouper avec plus d'intensité dans la réalité que ce qui pourrait être observé si les liens étaient distribués uniformément. La comparaison du clustering des deux graphes aléatoires conduit à la même conclusion pour les deux populations bien que la différence de clustering soit plus faible $(0,47$ contre 0,38$)$. La confrontation des valeurs prises par les indicateurs de distance conduisent à conclure que les administrateurs doivent tout de même parcourir une distance supérieure $(3,54)$, dans les faits, à ce qui seraient observable si les liens étaient uniformément distribués.

77 Si l'on compare le réseau observé à la projection unimode d'un réseau aléatoire biparti dont les distributions sont poissoniennes, le quotient $S W$ est égal à:

$(0,82 / 3,54) \times(2,20 / 0,38)=1,34$ 區 1 .

Ainsi, la propriété du petit monde observée sur le réseau des administrateurs de compagnies d'assurance est tout à fait normale. Elle provient de la structure même du réseau organisé en conseils. Elle resterait sans doute vérifiée dans la plupart des cas si l'on comparait une réalisation d'un graphe aléatoire biparti à un graphe aléatoire classique unimode.

L'analyse du réseau des administrateurs de compagnies d'assurance montre que le réseau aléatoire biparti équivalent en termes de distributions donne des estimations divergentes de celles du réseau réel. Le modèle sous-estime la mesure de clustering et de distance géodésique, indiquant que peut être certaines forces non aléatoires sont en oeuvre.

\section{Conclusion}

79 Cette étude porte sur la structure du réseau des administrateurs des compagnies d'assurance en 1881. Elle teste pour la première fois l'hypothèse du petit monde dans une perspective historique en mobilisant des indicateurs permettant d'appréhender l'architecture du réseau global. La structure d'un graphe petit monde est une structure intermédiaire entre celle d'un graphe de Caveman et celle d'un graphe dont les liens sont distribués de façon aléatoire. Elle est caractérisée par une distance géodésique proche de celle d'un graphe aléatoire. La valeur de son coefficient de clustering est toutefois, à l'instar du clustering d'une structure de type de Caveman, largement supérieure à celle d'un graphe aléatoire. Grâce à l'existence d'une faible proportion de " raccourcis ", c'està-dire d'administrateurs multi-mandats pour la dimension étudiée, l'information peut circuler rapidement à travers le réseau malgré l'existence d'un grand nombre de sommets. L'appartenance au réseau, et plus précisément au cœur du réseau que constitue la plus grande composante, permet aux administrateurs d'échanger des flux d'information. Les processus d'imitation peuvent se développer. L'importation de méthodes de gestion est facilitée.

80 L'étude montre que le partage d'administrateurs est une pratique ancienne puisqu'elle caractérise le réseau des administrateurs des principales compagnies d'assurance en France en 1881. L'existence de liens humains entre conseils aboutit à un réseau dont la structure est de type Petit Monde. Autrement dit, il ne faut que peu d'intermédiaires pour que deux administrateurs de ce réseau, quels qu'ils soient, se rencontrent malgré l'importance de la population. Nulle volonté particulière des uns ou des autres n'est 
nécessaire pour que le monde soit petit. Il suffit d'un faible pourcentage d'administrateurs aux mandats multiples se plaçant en intermédiaires privilégiés. En simulant plusieurs types de graphes aléatoires bipartis obtenus à partir de fonctions génératrices des distributions du nombre de mandats et de la taille des conseils, l'étude montre que la vérification de la propriété du petit monde découle de l'organisation du réseau sous forme de conseils d'administration. Même s'il existe des stratégies individuelles, elles ne remettent pas en cause cette propriété. En effet, les estimateurs du graphe aléatoire construit à partir des distributions empiriques divergent de ceux afférant à la population réelle. Ils sous-estiment à la fois le clustering et la distance géodésique, signe peut être que les administrateurs ont tendance à favoriser la présence de certaines de leurs relations dans les conseils; il est possible aussi que cette caractéristique résulte de la structure de groupe du secteur de l'assurance.

Ce travail permet d'envisager plusieurs pistes de recherches. En suivant le courant de recherche privilégiant la vision disciplinaire de la gouvernance, il procure des outils susceptibles d'accroître le pouvoir explicatif de modèles visant à appréhender la performance des entreprises, de mieux comprendre les stratégies d'enracinement des acteurs, d'améliorer la compréhension des divers moyens mis en œuvre pour lutter contre les comportements égocentriques. Dans un autre registre, il peut servir de support à des études portant sur la gouvernance cognitive dès lors qu'il s'agit de mieux cerner les processus cognitifs en œuvre au sein des conseils d'administrations, l'appropriation des connaissances, la construction des compétences, l'apprentissage des dirigeants. Les réseaux d'administrateurs peuvent aider le dirigeant à construire sa vision en la confrontant à leurs modèles mentaux. Ils leur permettent ainsi d'accéder à certaines des ressources nécessaires à l'élaboration de nouvelles stratégies.

\section{BIBLIOGRAPHIE}

Allen, M., 1974, “The structure of Interorganizational Elite Cooptation: Interlocking Corporate Directorates", American Sociological Review, 39, p. 393-406.

Bellon, B., 1980, Le pouvoir financier et l'industrie en France. Editions du Seuil, Paris.

Berle, A., Means, G., 1932, The modern corporation and private property, MacMillan, New-York.

Burt, R., 1983, Corporate Profits and Cooptation: Networks of Market Constraints and Directorate Ties in the American Economy. Academic press, New York.

Carrington, P., 1981, Horizontal co-optation through corporate interlocks, $\mathrm{PhD}$ thesis, Univ. Toronto.

Chabi, S., J. Maati, 2006, “The Small World of the CAC 40”, Banque et Marchés 82, pp. 41-53.

Charreaux, G., 2005, « Pour une gouvernance d'entreprise "comportementale", une réflexion exploratoire », Revue Française de Gestion, 157, p. 215-238.

Courtois, Alph. fils, 1883, Manuel des fonds publics et des sociétés par actions, $8^{\mathrm{e}}$ édition, Garnier frères, Paris 
Davis, G., M. Yoo, W. Baker, 2002, “The Network Topography of the American Corporate Elite, 1982-2001", Working paper, University of Michigan Business School.

DiMaggio P., W. Powell, 1983, “The Iron Cage Revisited: Institutional Isomorphism and Collective Rationality in Oganizational Fields. American Journal of Sociology, vol. 48.2, p. 147-160.

Hautcoeur, P-C., 2007, Le marché financier français au XIX siècle: Récit, Publications de la Sorbonne, Paris.

Kadushin, C., 1995, "Friendship among the French Financial Elite", American Sociological Review, 60.2 , p. 202-221.

Maati, J., 1998, "Le conseil d'administration: outil de contrôle et d'ordonnancement social des firmes en France", Conférence Internationale de l'AFFI.

Maati, J., 2008, “Le réseau des administrateurs européens: une application de la théorie du petit monde", Banque et Marchés 94, pp. 20-31.

Martin, P., 2005 « Les assureurs, des praticiens du risque ( $\mathrm{XIX}^{\mathrm{e}}-\mathrm{XX}^{\mathrm{e}}$ siècles) », Journée "Risque et économie", Association française des historiens économistes.

Milgram, S., 1967, “The Small World Problem”, Psychology Today, 2, p. 60-67.

Mills, C., 1956, The Power Elite. Oxford University Press, New York.

Mintz, B., M. Schwartz, 1981a, “Interlocking Directorates and Interest Group Formation”, American Sociological Review, 6, p. 851-68.

Mintz, B., M. Schwartz 1981b, “The Structure of Intercorporate Unity in American Business”, Social Problems, 29, p. 87-103.

Mizruchi, M., 1996, "What Do Interlocks Do? An Analysis, Critique, and Assessment of Research on Interlocking Directorates”, Annual Review of Sociology, 22, p. 271-298.

Mizruchi, M., S. Han, G. Dordick, 1995, « Flux de ressources et réseaux inter-organisationnels », Revue Française de Sociologie, 36, p. 655-684.

Morin, F., 1996, « Privatisation et dévolution des pouvoirs: le modèle français du gouvernement d'entreprise », Revue Economique, 6, p. 1 253-1 268.

Newman, M., S. Strogatz, D. Watts, 2001, "Random Graphs with Arbitrary Degree Distribution and their Applications", Physical Review, 64, p. 1-18.

North, D., 1990, Institutions, Institutional Change, and Economic Performance. Cambridge: Cambridge University Press.

Pennings, J.-M., 1980. Interlocking Directorates, Jossey-Bass, San Francisco.

Pfeffer, J., G. Salancik, 1978, The External Control of Organizations: a Ressource dependence perspective. Harper and Row, New York.

Radcliff, R., 1980, "Banks and Corporate Lending: an Analysis of the Impact of the Internal Structure of the Capitalist Class on the Lending Behavior of Banks", American Sociological Review, 45 , p. 553-570.

Rietsch, C., 2007 « Un indice des Compagnies d'assurance à la Bourse de Paris jusqu'en 1881 », 24 journées internationales d'économie monétaire, Rennes.

Rietsch, C., F. Duneau, 2007, « Les compagnies d'assurance sur le marché boursier français jusqu'en 1870 », pp. 507-550, in sous la direction de G. Gallais-Hamonno, Le marché financier français au XIX $X^{e}$ siècle, vol. II, Ed. de la Sorbonne, Paris. 
Siegel, S., 1956, Non parametric statistics for the behavioral sciences, McGraw- Hill

Scott, W., 2001, Institutions and Organizations. Thousand Oaks: Sage, 2nd edition.

Watts, D. 1999, "Networks, Dynamics, and the Small World Phenomenon", American Journal of Sociology, 105, p. 493-527.

Watts, D., et Strogatz, 1998, "Collective dynamics of small world networks", Nature, 393, p. 440-

442 .

Windolf, P., 1999, «L'évolution du capitalisme moderne - la France dans une perspective comparative », Revue Française de Sociologie, 43.

\section{NOTES}

1. Les particules ne constituent pas un signe obligatoire de noblesse mais le laissent miroiter dans l'œil du souscripteur.

2. Les résultats peuvent être fournis à la demande par les auteurs. L'analyse statistique des différences entre branches de risques et entre catégories d'administrateurs se base sur l'analyse de variance, d'autres méthodologies étant envisageables.

3. Une clique est un sous-graphe complet, un ensemble d'individus en contact étroit et assez fermé.

INDEX

Code JEL D85 - Network Formation and Analysis: Theory, Z13 - Economic Sociology • Economic Anthropology $\bullet$ Social and Economic Stratification

\section{AUTEURS}

\section{JÉRÔME MAATI}

Université de Lille 1, 104, avenue du peuple belge - 59043 Lille Cedex, jerome.maati[at]univlille1.fr

\section{CHRISTIAN RIETSCH}

Université d'Orléans BP 6739 - 45067 Orléans cedex 2 christian.rietsch[at]univ-orleans.fr 